\title{
Development of Effective Segment Erection System for Shield Machine
}

\author{
Shinichiro Aoe*, Tomoaki Sato*, Kenichi Murano**, and Shuichiro Nomura**
}

* Applied Technology Research Center, NKK Corporation, 1-1, Minamiwatarida-cho, Kawasaki-ku, Kawasaki, 210 JAPAN

** Steel Structure Machinery and Construction Division, NKK Corporation, 2-1, Suehirocho, Tsurumi-ku, Yokohama, 230 JAPAN

\begin{abstract}
Automatic segment assembly technology for use with the shield construction method has, as a result of the recent increase in the diameters of shield tunnels, become increasingly important as a way to improve safety and cut labor requirements at shield construction sites, and the development process has now passed from the research and development to the practical application stage. And there is now a greater demand than ever before for the function of the automatic assembly apparatus to reduce assembly time and provide fully automatic operation. For this reason, an assembly control method using information about contact between the segments has been developed along with an assembly algorithm that creates, on a computer, optimum assembly target data for several pieces to be assembled last based on assembly performance information of each segment. This report is an outline of the newly developed system.
\end{abstract}

\section{Introduction}

The tendencies seen in recent years for shield tunnels to be longer, have larger diameters, and be deeper than in the past and to be constructed more quickly than before have made improving efficiency, reducing labor requirements, and providing greater safety at shield construction sites increasingly important. Automatic segment assembly systems are to be introduced in order to guarantee safety in the face of expanding shield tunnel diameters and to overcome limits on what human workers can accomplish. But the use of automatic assembly systems has been hampered because it is difficult both to reduce assembly time and to automatically assemble a continuous series of segment rings.

To resolve these problems, an assembly control system which can detect the contact state between segments and, using a state transition graph based on a heuristic rule, can efficiently assemble the segments into the final contact state ${ }^{1)}$ has been developed along with an assembly algorithm that creates, on a computer, optimum assembly target data. 


\section{Assembly Control}

The contact state between work pieces is an important issue in the automation of assembly work. Improper contact can have several adverse effects: imprecise positioning, damage to segments, and excess reaction load on the machinery. A method to control assembly strategies through regulating the contact force has been developed to prevent these problems.

\subsection{Distinguishing the Contact State}

Figure 1 presents the shapes of a shield segment and its contact surface. The end face between the segments are provided with an inlaying pin-hole structure. Contact states are categorized by the ways in which pins and holes contact each other.

Categories of the contact states under this study were point - point, point - ridge line, point - plane, ridge line - ridge line, ridge line - plane, and plane - plane. Certain shapes of work pieces produce contact states that do not belong to any of these categories. In this study, it is assumed that they can be ignored and they do not generate any friction during contact.

When an assembly work based on the contact state is done manually, the contact state is judged and estimated from the configuration of the work pieces by a worker. This estimation is vague, as is the estimation of its state transition. Because a worker can learn the assembly work, the time required to perform assembly gradually shortens and the work precision rises. The assembly of somewhat complicated components is currently done manually because it can not be automated.

This development work was conducted on the assumption that it is acceptable to estimate the contact state vaguely, and its goal was an automated system that achieves higher speed and greater precision through a learning process. First, the contact state is roughly hypothesized and calculated. The contact state is hypothesized as 36 states for a total of 20 points - 16 points that form the inlaying pin-hole structure shown in Figure 1 and 4 points that form the ends of the work pieces - and the reaction force vectors to be measured in each contact state are calculated in advance ${ }^{2)}$. Next, the reaction force vectors calculated in advance are compared with the measured reaction force vectors, and the one that achieves the best fit is treated as the contact state at that time. The method applied here is to calculate the goodness-of-fit between the calculation and measurement results for 6 component forces (translation forces: $F_{x}, F_{y}, F_{z}$, moment: $M_{x}, M_{y}, M_{z}$ ) by using the membership function.

\section{$2.2 \quad$ State Transition Representation Method}

Because the movement vector can be calculated after the contact state is known, it is also possible to calculate the transition of the contact state. As the goal is the final assembled state, it is necessary to select the movement vector that can achieve the transition to that state.

Theoretically, if the movement vector is given and the movement is made in that direction, the next contact state is determined, but the transition is not always the one calculated, because the contact state itself is vague and the positioning mechanism is 
prone to play and flexure. For this reason, the state transition representation method proposed here is a method devised with reference to fuzzy automaton.

The present state is assumed to be $S_{i}(i=0, \ldots, n: n$ is the total number of states $+1, S_{0}$ is a non-contact state). When the probability of a transfer from $S_{i}$ to $S_{j}$ based on the movement vector $V_{i}$ is assumed to be $\left(a_{i j}\right)$, a transition graph matrix $A$ is created for each movement vector. $A$ is necessary for each movement vector $\boldsymbol{V}_{i}$.

The value of $\left(a_{i j}\right)$ is an index that provides the ease of the transition. It is provided by man in advance, and can be learned experimentally. If, for example, it is assumed that there is a high probability of a transition from $S_{i}$ to $S_{j}$ by a certain movement vector $\boldsymbol{V}_{i}$, but the transition that actually occurs is to $S_{k}$, the value is modified, giving a higher probability value to $\left(a_{i k}\right)$ than $\left(a_{i j}\right)$. After this modification, the search for an assembly control transition route is altered and a more realistic transition is selected, which will help shorten the assembly time.

The search for a route is performed by evaluating the cost of each route. The cost of a route is the sum of the weight of the transition frequency and the inverse number of the transition probability. The first movement vector that provides the cost-minimum route is the one to be selected.
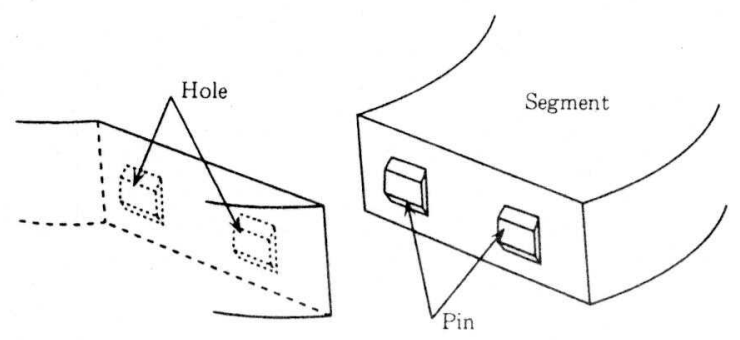

Figure 1 Inlaying Pin and Hole

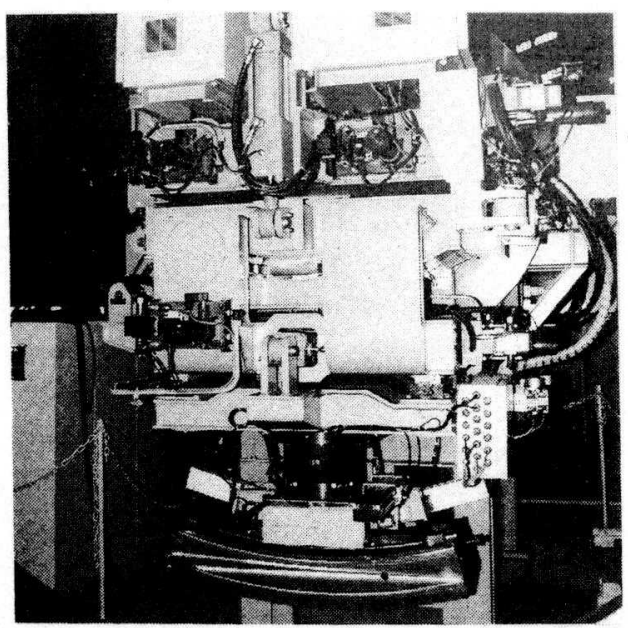

Photo 1 Experimental Automatic Erector

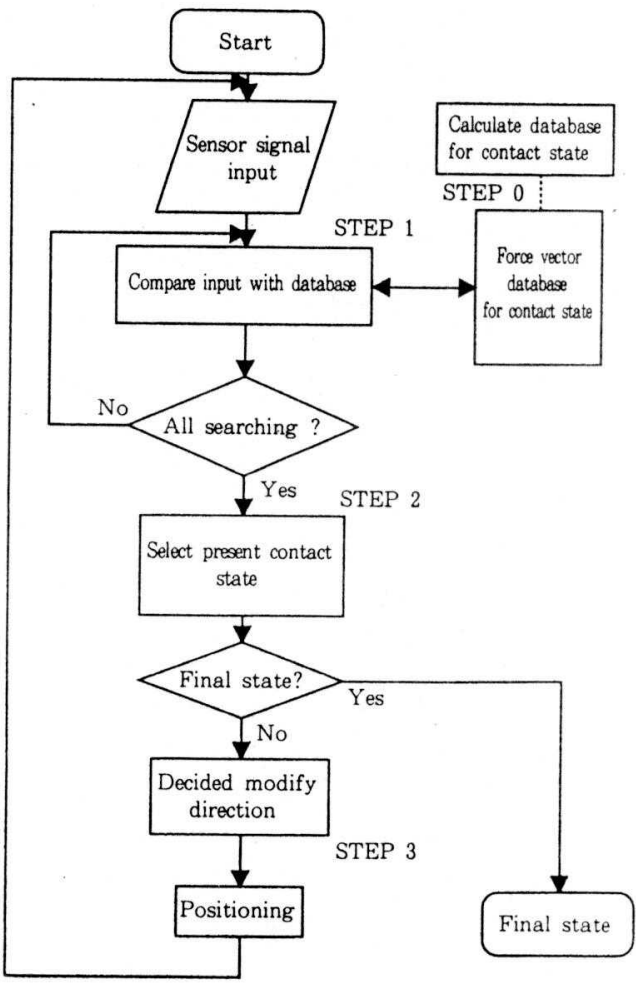

Figure 2 Flow Chart of Force Steering Positioning Control Algorithm 


\subsection{Control Algorithm}

Figure 2 shows the control algorithm. Each step is explained below.

Step 1 Force data to be measured by a force sensor for each contact state category is calculated, and stored in a data base.

Step 2 Position correction is done so that the transition is made toward the final state.

Step 3 When the contact is measured, the measured force data is compared with the data base. The contact state that corresponds to the force data with the best conformity is judged to be the present contact state.

Step 4 The transition graph is searched for position corrections that will permit a transfer to the final contact state. The movement vector that gives the shortest and cost-minimum transition route to the final state is selected, and the next position correction amount is determined accordingly.

Steps 2 to 4 are repeated until the final contact state is reached at Step 3, when the control operation is completed.

\subsection{Testing}

Testing was done with the assembly test equipment shown in Photograph 1 . The segments to be assembled had an external diameter of $4,036 \mathrm{~mm}$, a width of $400 \mathrm{~mm}$, and each weighed approximately $300 \mathrm{~kg}$. The mechanism was expressed by a rectangular coordinate system with the $x$ axis in the vertical direction and the $z$ axis in the tunnel excavation direction. It provided 8 degrees of freedom: the translation $z$ axis from the base axis, rotation $\theta$ around the $z$ axis, radial expansion $r$ axis, minute rotation $\theta_{x}, \theta_{y}$, $\theta_{z}$ around each axis, minute rotation $\Delta \theta_{z}$ around the $z$ axis, and minute translation $\Delta z$ along the $z$ axis. The two base axis $z, \theta$ are used for rough segment positioning, and are not used during fine positioning.

\subsubsection{Fitting Tests}

The distance between the segment already in place and another segment to be placed next was set at $10 \mathrm{~mm}$, and the algorithm shown in Figure 2 was applied to achieve the final contact state when the level difference is $1 \mathrm{~mm}$ and $2 \mathrm{~mm}$. The test results confirmed that it is possible to achieve a fit between the work pieces with precision of $0.3 \mathrm{~mm}$ by performing the transfers shown in Figure 3 for each initial level difference.

\subsubsection{Learning Tests}

Testing of the algorithm shown in Figure 2 revealed that according to circumstances, the results explained in the previous section were not obtained and the positioning did not converge. This is believed to be a result of the inability to escape from the deadlock state because of a deviation from the forecast transition route. Therefore, the transition results were studied, the transition matrix modified, the history of the transition state observed, and a heuristic rule that prevents a deadlock was added, with the result that a 


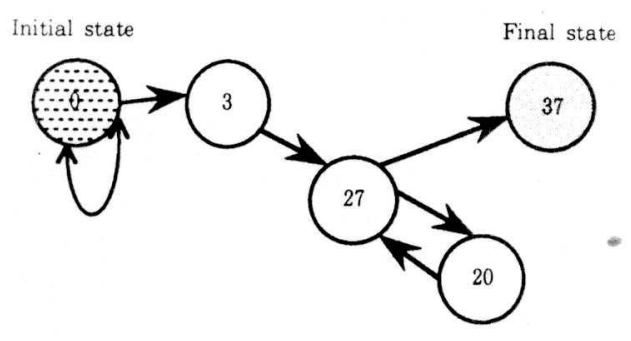

Transition arc in case of a $1 \mathrm{~mm}$ defference of level

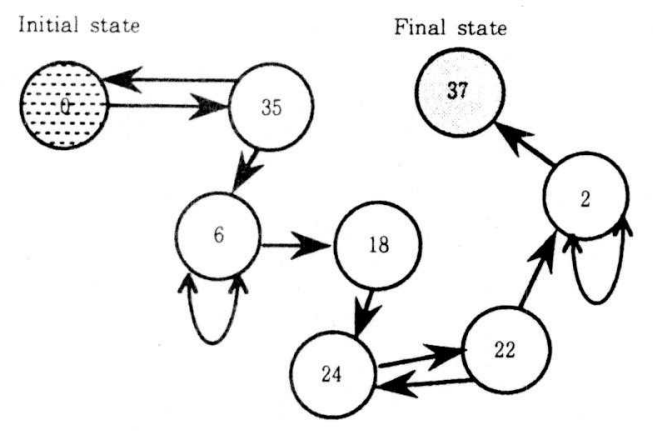

Transition arc in case of a $2 \mathrm{~mm}$ defference of level

Figure 3 Transition flow of inlaying experiment

deadlock no longer occurred, and it was confirmed that transition to the final state was achieved by means of transitions similar to the results shown in Figure 3.

\section{Segment Assembly Planning Method}

Automatic shield segment assembly methods in common use have involved fitting the interior surface edge of the segment to be placed to that of the segment already in place and positioning it in a way that minimizes the level difference between the segments ${ }^{3)}$ (Profile Segment Assembly Method (PSAM)). However, it was found that it was difficult to perform automatic assembly continuously using this method because of flattening of segment positioning errors and flattening of the segment rings by soil pressure.

This paper proposes Automatic Segment Assembly Algorithm (ASAA). ASAA has two basic algorithms. One of the algorithms makes the segment ring being installed as close to a complete round as possible compared to the flattened ring already in place. The other algorithm permits forecasting the space required for the $\mathrm{K}$ segment in order to achieve optimum assembly of the segment ring. This paper also proposes a segment assembly simulator of ASAA.

\subsection{Automatic Segment Assembly Algorithm (ASAA)}

Figure 4 shows a model of a segment. Figure 5 shows a segment ring formed by segments. A segment is one of the several pieces into which a ring is divided and is made of reinforced concrete. It comes with a number of bolt holes formed so that the ring already in place can be connected to the next segment. As shown in Figure 5, the segments of a ring are defined as $\mathrm{A}_{1}, \mathrm{~A}_{2}, \mathrm{~A}_{3}, \mathrm{~A}_{4}, \mathrm{~A}_{5}, \mathrm{~A}_{6}, \mathrm{~A}_{7}, \mathrm{~A}_{8}, \mathrm{~B}_{1}, \mathrm{~B}_{2}$, and $\mathrm{K}$. The segments are installed in this sequence. The segments from $A_{1}$ to $A_{8}$ are defined as the A segments. Segments $B_{1}$ and $B_{2}$ are defined as the $B$ segments. Figure 6 presents a flow chart of ASAA. The following is an explanation of these steps.

\section{Step 1 Measurement of the ring already placed}



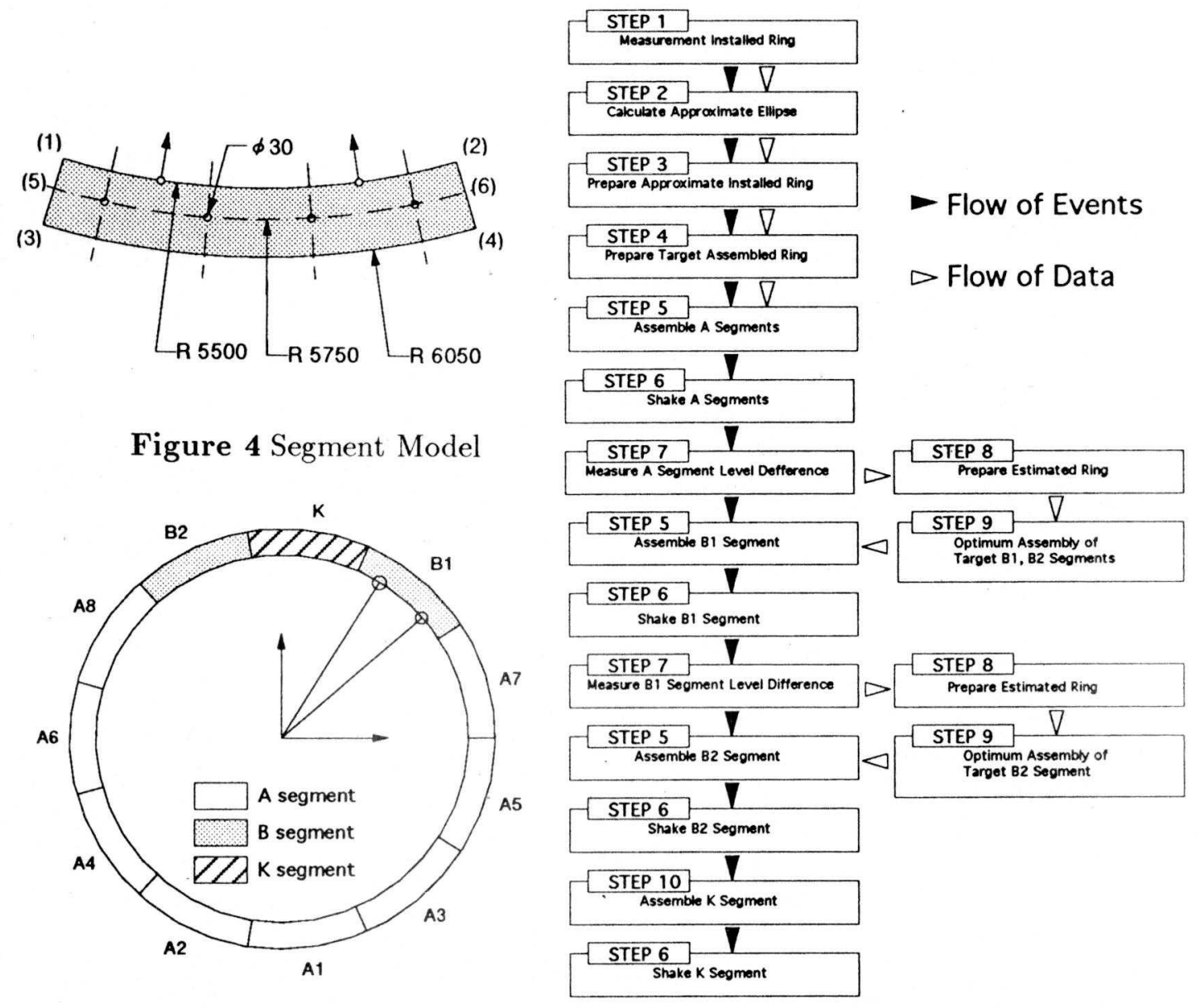

Figure 5 Segment Ring

Figure 6 ASAA Flow Chart

As shown in Figure 5, the interior surface of the ring is measured. The position data at two points of each segment $\left(X_{i}^{M}, Y_{i}^{M}\right)(i=1, \ldots, 22)$ is measured.

\section{Step 2 Calculation of Approximate Installed Ellipse}

$\left(X_{i}^{M}, Y_{i}^{M}\right)$ obtained at Step 1 is used to calculate the parameters of the approximate installed ellipse (major axis $a$, minor axis $b$, tilt $\phi$, center point $\left(X_{c}, Y_{c}\right)$ ) based on the segment ellipse calculation method (SECM).

\section{Segment Ellipse Calculation Method (SECM)}

$\left(X_{c}, Y_{c}\right)$ is found as the center of gravity of the 22-polygon formed by $\left(X_{i}^{M}, Y_{i}^{M}\right)$. $a, b$, and $\phi$ are found from the coefficients of the equation of an ellipse which has $\left(X_{c}, Y_{c}\right)$ using the least square method from $\left(X_{i}^{M}, Y_{i}^{M}\right)$.

\section{Step 3 Preparation of Approximate Installed Ring}

The segment ring $E$ is prepared from the approximate installed ellipse calculated at Step 2, using the segment ellipse assembly method (SEAM). From $\left(X_{i}^{M}, Y_{i}^{M}\right)$ obtained at Step 1 and the positions of the $\operatorname{ring} E\left(X_{i}^{E}, Y_{i}^{E}\right)(i=$ 
$1, \ldots, 22)$ corresponding to $\left(X_{i}^{M}, Y_{i}^{M}\right)$, the following formulae are used to prepare the positions $\left(X_{i}^{A}, Y_{i}^{A}\right)(i=1, \ldots, 22)$ of the approximate installed ring $A$ that corresponds to $\left(X_{i}^{M}, Y_{i}^{M}\right)$.

$$
\begin{aligned}
& X_{i}^{A}=0.5 X_{i}^{M}+0.5 X_{i}^{E} \\
& Y_{i}^{A}=0.5 Y_{i}^{M}+0.5 Y_{i}^{E}
\end{aligned}
$$

$\left(X_{i}^{A}, Y_{i}^{A}\right)$ are used to prepare the interior surface curve of the approximate installed ring.

\section{Segment Ellipse Assembly Method (SEAM)}

It is hypothesized that the position of the segment is represented by the opening angle $\Delta \theta_{i}$ between the segment $i$ and segment $i+1$. SEAM is based on the annular beam bending theory ${ }^{4)}$. The theory of the deformation of an annular beam when a certain load acts upon it is applied to a segment ring. If it is assumed that $\Delta \theta_{i}$ corresponds to the bending moment of the annular beam subjected to a tensile load in a certain direction, $\Delta \theta_{i}$ is represented by an approximate expression as follows.

$$
\Delta \theta_{i}=\sum_{n=1}^{2} D_{n}\left\{\frac{\sum_{j=1}^{11}\left|\cos \left(\hat{\theta}_{j}-\phi\right)\right|^{2 n-1}}{11}-\left|\cos \left(\hat{\theta}_{i}-\phi\right)\right|^{2 n-1}\right\}
$$

where, $\phi$ is the direction of the tensile load, $\hat{\theta}_{i}$ is the angle of inclination of the segment $i$ in the complete round segment ring, and $D_{1}, D_{2}$ express values corresponding to the tensile load.

When the segment ring is found from given ellipse parameters, $D_{1}, D_{2}$, and $\phi$ are found from the data base and these are used to find the segment ring.

\section{Step 4 Preparation of Target Assembled Ring}

From the approximate installed ellipse parameters obtained at Step 2, the parameters (major axis $\hat{a}$, minor axis $\hat{b}$, tilt $\hat{\phi}$, and center point $\left.\left(\hat{X}_{c}, \hat{Y}_{c}\right)\right)$ of the target ellipse which is directed somewhat closer to the complete round and the center point are prepared. SEAM is used to prepare the target assembled ring $O$ from the target ellipse.

\section{Step 5 Segment (A and B Segments) Assembly}

The segment to be installed is pushed against the segment previously installed using the assembly control method described in Chapter 2 in such a way that $\Delta \theta$ and the level difference $\Delta x$ are 0 (in order to compress the sealant). As shown in Figure 7 , the objective function $f_{\text {obj }}$ is used to assess the level difference between the existing ring and the segment being installed. $\Delta \theta$ which minimizes $f_{\text {obj }}$ is determined, and each segment is assembled accordingly.

$$
f_{\text {obj }}=\left|1.5\left(\Delta P_{2}-\Delta \tilde{P}_{2}\right)-0.5\left(\Delta P_{1}-\Delta \tilde{P}_{1}\right)\right|
$$

\section{Step 6 Shaking the Segment}


When the bolt insertion condition has not been satisfied after a segment has been assembled, the segment is shaken. Here, this condition means that the distance between the center points of the bolt holes on the segment that has already been installed and on the segment to be installed is within $2.5 \mathrm{~mm}$. If this condition is not satisfied, it is impossible to fix the segment in place, and the automatic assembly is suspended.

\section{Step 7 Measurement of Segment Level Difference after Assembly}

Measurements are made of $\Delta P_{1}, \Delta P_{2}$, and $\Delta x$ between the assembled segment and the ring installed previously.

\section{Step 8 Preparation of Estimated Segment (A and B segments)}

The segment assembled at Step 6 is estimated with reference to the interior surface curve of the approximate installed ring $A$ so that $\Delta P_{1}, \Delta P_{2}$, and $\Delta x$ are as identical as possible with those value measured at Step 7 . The segment which has been estimated in this way is defined as the estimated segment.

\section{Step 9 Optimum Assembly Planning of Target B Segments}

As shown in Figure 8, optimum assembly planning for the target B segments is performed based on the estimated ring from Step 8 in order to provide assembly space for the $\mathrm{K}$ segment.

\section{Optimum Assembly Planning of Target $B_{1}$ and $\mathbf{B}_{2}$ Segments}

The function $f_{\mathbf{s}}$ described below is applied to assess the $\mathrm{K}$ segment assembly space based on the estimated ring which consists of the estimated A segments.

$$
f_{\mathrm{s}}=\min \left(\Delta d_{12}, \Delta d_{34}, \Delta d_{14}, \Delta d_{23}\right)
$$

where,

$\Delta d_{12}:$ Difference between distance $\left[\mathrm{B}_{1}(2), \mathrm{B}_{2}(1)\right]$ and the distance $[\mathrm{K}(1), \mathrm{K}(2)]$

$\Delta d_{34}$ : Difference between distance $\left[\mathrm{B}_{1}(4), \mathrm{B}_{2}(3)\right]$ and the distance $[\mathrm{K}(3), \mathrm{K}(4)]$

$\Delta d_{14}$ : Difference between distance $\left[\mathrm{B}_{1}(4), \mathrm{B}_{2}(1)\right]$ and the distance $[\mathrm{K}(1), \mathrm{K}(4)]$

$\Delta d_{23}$ : Difference between distance $\left[\mathrm{B}_{1}(2), \mathrm{B}_{2}(3)\right]$ and the distance $[\mathrm{K}(2), \mathrm{K}(3)]$

$\Delta \theta_{\mathrm{B} 1}, \Delta \theta_{\mathrm{B} 2}$ are set in such a way as to minimize the following objective function.

$$
f_{\mathrm{obj}}=\max \left(0.4 d_{\mathrm{M}}\left|\Delta \theta_{\mathrm{B} 1}\right|, 0.4 d_{\mathrm{M}}\left|\Delta \theta_{\mathrm{B} 2}\right|,\left|f_{\mathrm{s}}\right|\right)
$$

where, $d_{\mathrm{M}}$ is distance between the center points of the segment ends. The estimated ring and $\Delta \theta_{\mathrm{B} 1}, \Delta \theta_{\mathrm{B} 2}$ are used to prepare the target $\mathrm{B}_{1}$ and $\mathrm{B}_{2}$ segment.

\section{Optimum Assembly Planning of Target $\mathrm{B}_{2}$ Segment}

The estimated ring which consists of the estimated $\mathrm{A}$ segments and the estimated $B_{1}$ segment are used to determine $\Delta \theta_{\mathrm{B} 2}$ in such a way that the following objective function is minimized.

$$
f_{\text {obj }}=\max \left(K_{\mathrm{B}} d_{\mathrm{M}}\left|\Delta \theta_{\mathrm{B} 2}\right|,\left|f_{\mathrm{s}}\right|\right)
$$

The estimated ring and $\Delta \theta_{\mathrm{B} 2}$ are used to prepare the target $\mathrm{B}_{2}$ segment. 

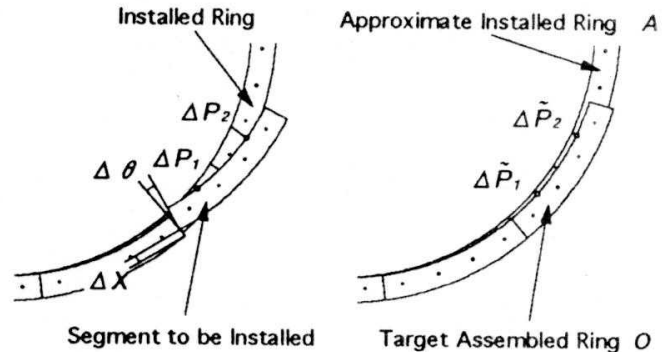

Target Assembled Ring $O$

Figure 7 Assembly of Segments

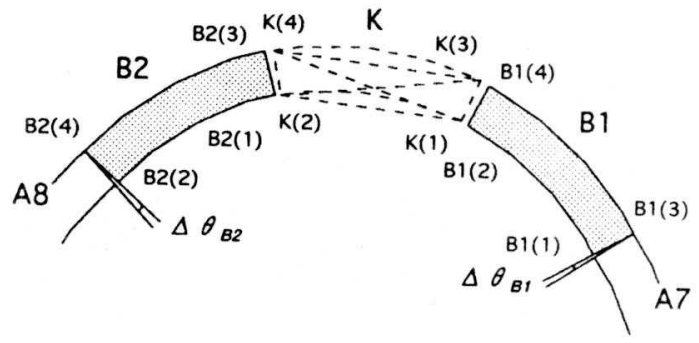

Figure 8 Optimum Assembly Plan for Target B Segments

Table 1 Simulation Conditions

\begin{tabular}{|l||l|}
\hline Tunnel Inside Diameter & $11,000 \mathrm{~mm}$ \\
\hline Number of Segments & 11 \\
\hline Number of Bolt Holes & 4 \\
\hline Bolt Hole Diameter & $30 \mathrm{~mm}$ \\
\hline Bolt Diameter & $25 \mathrm{~mm}$ \\
\hline Allowed Discrepancy Between Bolts & within $2.5 \mathrm{~mm}$ \\
\hline
\end{tabular}

\begin{tabular}{|l||l|}
\hline Initial Ring Oblateness (ASAA) & $0.2 \%$ \\
\hline Initial Ring Oblateness (PSAM) & $0.1 \%$ \\
\hline Ring Measurement Error & $\pm 1.0 \mathrm{~mm}$ \\
\hline Squeezing Error & $\pm 0.3 \mathrm{~mm}$ \\
\hline Level Difference Measurement Error & $\pm 0.05 \mathrm{~mm}$ \\
\hline Assembly Error & $\pm 0.2 \mathrm{~mm}$ \\
\hline
\end{tabular}

\section{Step 10 Segment Assembly (K Segment)}

The $\mathrm{K}$ segment is assembled in such a way that the level difference and gap between the $\mathrm{K}$ segment and the $\mathrm{B}$ segment.

\subsection{Automatic Segment Assembly Simulation}

In order to confirm the usefulness of ASAA, computer simulations were performed same as an actual automatic segment assembly system. When 100 rings had been assembled, the simulation was completed. When bolt insertion conditions were not satisfied, the simulation was suspended. Table 1 shows the conditions for the simulation.

The results of the simulation are presented in Figure 9 and Figure 10. The solid lines represent the results of the simulation of ASAA; the broken lines represents the results of the simulation of PSAM. Figure 9 represents the resulting oblateness $S_{1}$ of the assembled rings. When $S_{1}$ neared $0 \%$, the roundness of the rings was corrected. Figure 10 shows the resulting amount of deviation $S_{2}$ from the correct amount of assembly space for the $\mathrm{K}$ segment. If $S_{2}$ is equal to 0 , the $\mathrm{K}$ segment can be assembled without leaving any gap. The smaller the amplitude of $S_{2}$, the more stable the K segment assembly space.

In PSAM simulation, it became impossible to continue assembly after 13 rings were assembled. The results of ASAA simulation revealed that it was possible to assemble at least 100 rings. Figure 9 shows that the initial $S_{1}$ of $0.2 \%$ gradually approached $0 \%$, the perfect roundness. In Figure 10, the amplitude of $S_{2}$ was about $2.5 \mathrm{~mm}$, a relatively stable level. The above results clearly illustrate that using ASAA, it is possible to perform continuous stable segment assembly for extremely long periods of time, even under conditions that tend to flatten rings. 


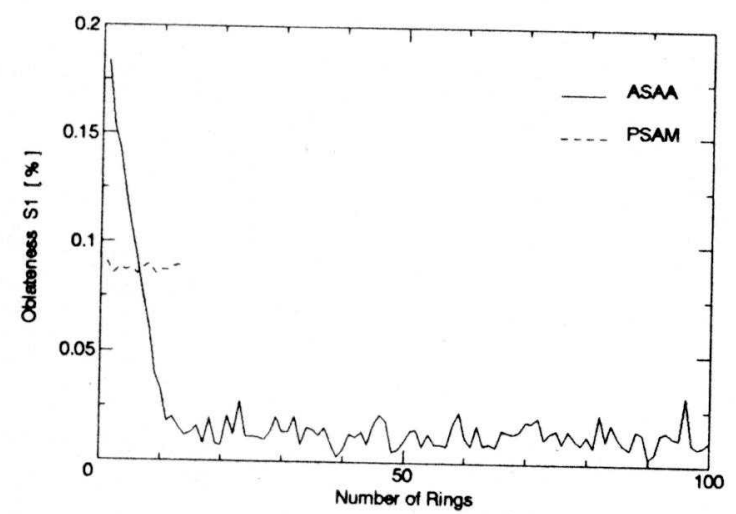

Figure 9 Simulation Results $\left(S_{1}\right)$

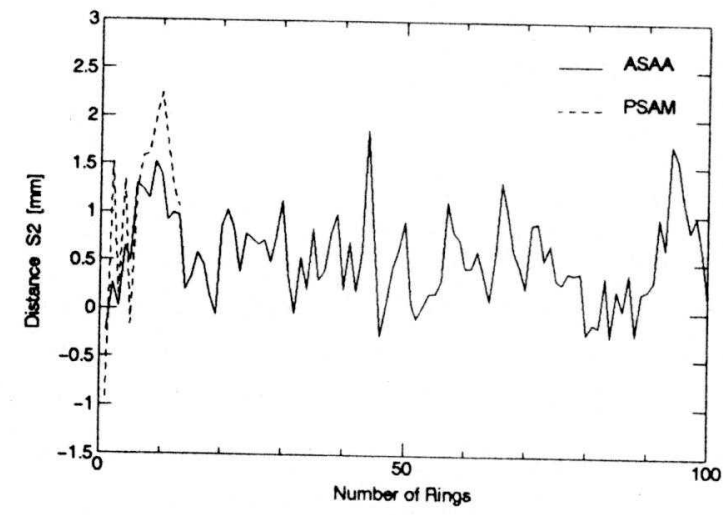

Figure 10 Simulation Results $\left(S_{2}\right)$

\section{Conclusion}

This work, conducted to provide one of the elemental technologies needed to develop an automatic segment assembly system for use with large diameter shield machines, is related to both assembly control and assembly planning methods. In addition to these, work has already been completed on a space-efficient erector mechanism ${ }^{5), 6)}$, a relative position measurement method that increases speed, and a bolt tightening apparatus that fully automates the bolt and nut stocking, supply, and tightening steps.

Future studies are expected to be focused on ways to improve overall system reliability, cut costs, and to apply the system to actual construction work.

\section{Reference}

1) Sato T. et al, "A Control for Building Shield Segments Using a Transition of Contact Status," The 73rd JSME Fall Annual Meeting, Vol. 5, pp. 249-250, 1995. (In Japanese)

2) Hirukawa H. et al, "Representation and Analysis of Constraints for Motions of Objects in Assembly Processes," Journal of the Robotics Society of Japan, Vol. 11, No. 2, pp. 192-200, 1993. (In Japanese)

3) Matsushita T. et al, "Development of Segment Automatic Building Intelligent System (SABIS)," 7th ISARC, Bristol England, Jun. 1990.

4) Shuji T., "Modern Material Mechanics," Ohm-sha, pp. 165-174, 1970. (In Japanese)

5) Murano K. et al, "The Parallel Mechanism of a Segment Automatic Erector," Proceedings of the 12th annual conference of RSJ, No. 3, pp. 809-810, 1994. (In Japanese)

6) Honbashi K. et al, "An Automatic Building System for Large Tunnel Segment," NKK Technical Report, No. 150, pp. 51-55, 1995. (In Japanese) 